IV.

Aus dem allgemeinen öffentlichen Krankenhause in Mährisch Rothwasser.

\title{
Mitteilung über einen durch Operation geheilten Fall von Thrombose im Gebiete der Vena mesenterica superior. Ton
}

\author{
Dr. Theodor Haagn, \\ Primararzt.
}

Wenn auch die Thrombose der Mesenterialgefäße überhaupt zu den seltensten Erkrankungen gehört, so würde ich dennoch zögern, die Literatur des durch und durch gut studierten Krankheitsbildes noch durch die Mitteilung eines weiteren Falles zu bereichern; allein die äußerst schlechte Prognose dieses Leidens, sowohl bei interner als anch chirurgischer Behandlung, läßt es gerechtfertigt erscheinen, jeden geheilten Fall zur Veröffentlichung zu bringen, um so mehr als der unregelmäßige Heilungsverlauf in unserem Falle mit schließlicher, endgültiger Genesung gewiß auch der Besprechung wert erscheint.

In dem von mir operierten Falle handelt es sich um eine 37jährige Frau, welche am 9. I. 1907 in das hiesige Krankenhaus aufgenommen wurde. Die Krankengeschichte ist folgende:

Anamnese: Die Frau hat 5 Geburten mitgemacht; die letzte vor $1^{11}$. Jahren. Damals bekam sie schweres Wochenbettfeber; der behandelnde Arzt konstatierto ,linksseitige Beckenbindegewebsentziundung". Die Frau war $1 / 2$ Jahr krank. Am Tage der Aufnahme 9. I. 7 Uhr früh traten plötzlich ohne jede Ursache heftige Schmerzen im Leibe auf, verbunden mit mehrmaligem Erbrechen, kein Abgang von Winden. Der herbeigerufene Arzt verordnete ein leichtes Abführmittel, worauf zwei breiige Stühle erfolgten. Die Schmerzen wollten trotz Injektionen (nach Mitteilung des Kollegen $2 \times 0,02$ Morphin) nicht weichen. Beim Untersuchen habe sich links eine äuRerst schmerzhafte Stelle gefunden. Die Frau gibt 
nun an, daß sie wahrend dieser Manipnlationen plötzlich einen Ruck Ferspürt hätte, worauf die Schmerzen nachlieben. „Es sei das Hindernis beseitigt worden", wie sie sagte.

Status praesens bei der Aufnahme 7 Uhr abends: Patientin macht einen schwerkranken Eindruck; Erbrechen besteht jedoch nicht mehr, ilagegen leichtes Aufstoßen. Abendtemperatur: 37,5, Puls 80.

Das Abdomen ist nur wenig aufgetrieben, links rom Nabel fühlt man eine wurstförmige, auf Berührung außerst schmerzhafte Resistenz. Patientin hat Stuhldrang, sie entleert etwa 1 Liter rein blutiger Flüssigkeit. Da der Puls kräftig ist und auch die bedrohlichen Erscheinungen anscheinend im Rückgange sind, wird zunachst eine abwartende Haltung eingenommen. Behandlung: Tinct. opii 3 stündlich 15 bis 20 Tropfen, feuchtwarme Umschläge am Leib.

10. I. Vormittagstemperatur 36,5 , Puls 90 , Schmerzen mäiig, Druckempfindlichkeit geringer. Durch Palpation läßt sich auch heute die vorher erwähnte Resistenz, die aber beweglicher erscheint, nachweisen; ferner besteht linksseitiger freier Leistenbruch.

Nachmittagstemperatur 38, Puls 100 , ahends $7 \mathrm{Uhr} 110$. Die vorgenommene Untersuchung ergibt: Meteorismus bedeutend stärker, die ganze linke Bauchseite druckemptindlich. Auf Einlauf entleeren sich 2 mal wieder rein blutige Stühle, mit der Klysma-Flüssigkeit etwa 2 bis 3 Liter.

Da die peritonealen Symptome im Ansteigen begriffen sind, wird am 11. I. zur Laparotomie geschritten, Medianschnitt bis $1 \mathrm{~cm}$ über dem Nabel; es treten sofort geblähte Darmschlingen hervor. Zwischen denselben quillt aus der Bauchhöhle braunrote Flüssigkeit heraus, die ausgeschöpft wird.

Die eingehende Hand fühlt links eine dicke wurstförmige Geschwulst, die vor die Bauchwunde gelagert wird und sich als ein Teil des Jejunums erweist. In einer Länge, von etwa $20-30 \mathrm{~cm}$ erscheint die Darmwand blaurot verfärbt, starr und etwa auf das 2 bis 3 fache verdickt. Das dazu gehörige Mesenterium zeigt bis zur Wurzel zahlreiche Thromben; das von denselben angefüllte Gekrösestück bildet ein Dreieck, dessen Basis das absterbende Darmstück, dessen Spitze die Radix mesenterii bildet. Die Darmserosa ist nicht glänzend, sondern erscheint matt und vielfach mit Fibringerinsel bedeckt. Vom aufsteigenden Colon zieht ein fester Strang zum Mesenterialansatz des oben erwähnten Darmteiles.

Der Strang wird ligiert und abgeschnitten. Der erkrankte Darn wird, nachdem das veränderte Gekröse in Dreieckform reseziert wurde, in einer Ausdehnung von $40 \mathrm{~cm}$ im Gesunden abgetragen, die Enden blind geschlossen und nun die seitliche Anastomose mittels Murphyknopf ausgefuhhrt. Hierbei läBt es sich nicht vermeiden, daß aus dem stark geblähten zentralen Darme Inhalt herausspritzt. Die beschmutzte Partie wird abgetupft und mit Kochsalzlösung gewaschen. Da aber einerseits durch die Thrombosierung eine verminderte Ernährung gefürchtet wird, andererseits der Austritt von Darminhalt eine Infektion nicht ausschließen 
läßt, wird drainiert, im übrigen die Bauchwunde wie üblich geschlossen. Anstieg der Temperatur nachmittags bis 39, Puls abends 100.

12. I. Pat. fühlt sich schwach, die peritonealen Erscheinungen jelloch geringer; Frühtemperatur 39,1, abends 38,5, Puls 100.

15. I. Temperatur 37,6, nachmittags 38,0 . Verbandwechsel, Heilung rer Hautwunde p. pr. Die Drainage wird gelockert. Puls 98.

16. I.-21. I. Temperaturen normal zwischen 36,0 und 37,2. Stuhl hat sich auf Glyzerinklysma eingestellt. Murphyknopf ging noch nicht ab.

22. I. Plötzlicher Anstieg der Temperatur bis 38,7. Verbandwechsel. Nach Entfernung der Drainage zeigt sich, daß sich eine Darmfistel gehillet hat, die gelbliches breiiges Sekret liefert.

Eutfieberung in den nachsten Tagen, Pat. vom 24. bis 28. fieberfrei.

29. I. Temperatur früh 37,7 , abends 39 .

30. I. Temperatur früh 37,2, nachmittags 37,5. Gegen Abend IleusErscheinungen, Erbrechen fajkulent riechender Massen, Puls 110.

31. I. Relaparotomis. Schnitt unterhalb des früheren, links vom Nabel. Nach Eröffnung des Abdomens sieht man mehrere Darmschlingen miteinander verwachsen; eine Schlinge erscheint spitzwinklig geknickt, der zuführende Teil geblaht. Es wird versucht, die Verwachsung zu lösen, jedoch reiBt hierbei die Darmwand wiederholt ein. Nach Vernähung derselben wird das zuführende Darmstück so weit als möglich freigemacht und unter leichten Zug in die Bauchwunde zwecks Ausführung der Enterostomie eingenäht. Die vorher erwähte Darmfistel ist nur dünn und wird in Anbetracht der Schwäche der Patientin auf den VerschluB derselben verzichtet, aus demselben Grunde anch auf eine Enteroanastomose. Der Murphyknopf ist nicht mehr an seiner Stelle.

1. II.-6. II. Langsame Entfieberung, Puls schwankt zwischen 98 und 100. Ileus-Erscheinungen verschwunden, die Enterostomie-Wunde hat sich geschlossen.

10. II. Temperatursteigerung 38.5. Auf Einlauf wenig Stuhl. Entficberung in den nächsten 3 Tagen.

14. II. Temperatur 38, nachmittags 37,5 .

15. IJ. Anstieg bis 39 ; derselbe ist bedingt durch Koprostase in den untersten Darmpartien. Am selben Tage Abgang des Murphyknopfes.

16. II. Untersuchung per rectum ergibt, daß der Mastdarm mit harten Kotballen ausgefüllt ist; digitale Entfernung derselben. Nun gelingt es durch regelmäBige hohe Einläufe mit 01 und Wasser eine gründliche Entleerung der tieferen Darmpartien herbeizuführen. Die Darmfistel hat sich spontan geschlossen.

19. II.-28. II. Pat. ist fieberfrei, Appetit stellt sich ein, die Frau erholt sich rasch.

28. II. Entlassung. Die Bauchwunden vollständig geschlossen, Narbe fest.

Nachuntersuchung Mitte Mai 1907 ergibt: Pat. hat sich bedeutend erholt, gibt an, regelmaßig jeden 2. Tag Stuhl zu haben. Der Appetit sei besser als vorher, auch vertrage sie die schwer verdaulichsten Speisen. Untersuchung des Leibes ergibt normale Verhältnisse.

15. VIII. Pat. ist vollständig hergestellt und kann wieder jede körperliche Arbeit verrichten. 
Die erste Frage, die wir uns im vorliegenden Falle zu beantworten haben, ist wohl die: Konnten wir auf Grund der bestehenden Symptome die bei der Operation gemachte Diagnose auch schon vorher stellen oder nicht?

Der plötzliche Beginn des Leidens mit Erbrechen, vollständiger Windverhaltung ließ natürlich zunächst an Ileus und zwar an Strangulations-Ileus denken. Auf Einlauf stellte sich breiiger Stuhl ein, was allerdings auch bei vollständiger Okklusion möglich ist. Der herbeigerufene Arzt konstatierte geringen Meteorismus, sowie links vom Nabel eine druckempfindliche Resistenz. Dieses anfänglich äußerst bedrohliche Bild besserte sich in den Nachmittagsstunden. Die Schmerzen wurden geringer, ja die Patientin glaubte, daß durch die medikamentöse Behandlung, sowie durch die äußere Untersuchung sich das Hindernis gelöst habe. Bei der Aufnahme besteht nur melır Aufstoßen; der Einlauf fördert massenhaft blutigen Stuhl zutage.

Damit mußte die Diagnose Strangulations-Ileus verlassen werden. Es kam weiterhin die Invagination in Betracht. Dagegen sprach vor allem das Alter der Patientin, da die Invagination fast ausschließlich eine Erkrankung der ersten Lebensjalre ist. Immerhin ist dieselbe auch bei Erwachsenen nicht allzu selten, jedenfalls viel häufiger als die Thrombose der Mesenterialgefäße. Ich entschloß mich daher zur Diagnose Intussuszeption um so leichter, als ja Darmblutung bei bestehenden Ileus-Erscheinungen das Hauptsymptom dieses Leidens darstellt. Die Operation klärte uns über den wahren Sachverhalt auf.

Welche Symptome gehören zum Bilde der "Thrombose der Mesenterialgefäße"? Kußmaul verlangt hierfür: 1. Nachweis der Quelle der Embolie. 2. Reichliche Darmblutung. 3. Sinken der Temperatur. 4. Peritoneale Symptome, wie Erbrechen, Bauchschmerz. Am konstantesten findet sich bei den akut auftretenden Fällen das Erbrechen; auch in unserer Krankheitsgeschichte steht dieses Symptom neben heftigen Leibschmerzen im Vordergrunde der Erscheinungen. Im Gegensatz hierzu ist manchmal der Beginn nicht so erschreckend; die Patienten leiden Tage hindurch an Diarrhöen, oder die Ileus-Erscheinungen sind nur schwach angedentet, nehmen langsam zu, wie es uns z. B. Reitzenstein in seinem Falle schildert; derselbe Autor macht auch auf einen Umstand aufmerksam, den ich bei Durchsicht der Literatur manchmal vorfand, ohne daß er besonders hervorgehoben wurde. Und doch scheint er mir gegenüber dem Strangulations-Ileus, besonders wenn die Blutung 
fehlt, für die Unterscheidung der beiden Krankheitsbilder von besonderer Wichtigkeit: es ist dies das plötzliche Verschwinden der schweren peritonealen Symptome. Auch bei meinem Falle entsprach der Zustand bei der Aufnahme, das ist 12 Stunden nach Beginn, nicht mehr dem Anfangsstadium. Das Erbrechen hatte aufgehört, anch der Meteorismus war nicht mehr derart, wie man ihn bei Ileus vorfindet, ebensowenig konnte man eine ausgesprochene Darmsteifung nachweisen.

Dagegen fand sich links vom Nabel eine wurstförmige Resistenz, die in Verbindung mit der bestehenden Darmblutung mich in der Diagnose Invagination noch bestärken mußte. Dieser Nachweis der Resistenz ist auch in vielen Fällen von Thrombose der Mesenterialgefäße zu erbringen, und hat derselbe insofern eine $\mathrm{Be}$ deutung, als man dadurch im Falle chirurgischen Eingreifens einen Anhaltspunkt für rie Stelle der Eröffnung der Bauchhöhle hat, während man bei Ileus wohl ausnahmslos in der Medianlinie eingehen muß. Wenigstens in meinem Falle entsprach die Resistenz genau dem infarcierten Darmstück. Natürlich ist dieses Symptom nur dann vorhanden, wenn der Krankheitsprozeß sich nur auf einen kurzen Darmteil beschränkt, und wenn der Meteorismus nur gering ist. Die Bedeutung dieses Symptomes für unser ärztliches Handeln wird bei Besprechung der Therapie erläutert werden.

Das zweite in die Augen springende Symptom war die auf Einlauf erfolgte Blutabsonderung ans dem Darme. Nun ist aber gerade die Blutung für die Diagnose der Venenthrombose von besonderer Wichtigkeit. Demgegenüber hat Borszeky darauf aufmerksam gemacht, daß dieses Symptom durchaus nicht so konstant ist, wie z. B. Kußmaul annahm. Unter 49 Fällen von Embolie der Mesenterialgefäße konnte er nur 14 mal Darmblitungen nachweisen.

Wann es zur Blutung bei diesem Leiden kommt und wann nicht, dafür gibt uns Sprengel eine Erklärung. Dieser Autor nnterscheidet nämlich scharf zwischen "hämorrhagischem Infarkt" und „anämischer Gangrän". Ersterer entsteht, wenn nur Arterie oder Vene allein verstopft sind; die Wirkung der Verstopfung von Arterie oder Vene ist nämlich nach Mikulicz und Nothnagel gleich; in beiden Fällen kommt es zum hämorrhagischen Infarkt. Anders bei der ischämischen Gangrän; dieselbe kommt dadurch zustande, daß Vene und Arterie verstopft sind. Diese Auffassung erfährt eine Ergänzung durch die Versuche Kaders, der fand, daß bei schwacher Strangulation des Darmes, wodurch 
nur der venöse Abfluß gehemmt wird, sich hämorrhagischer Infarkt entwickelt, während bei starker Strangulation, wobei Arterien und Venen komprimiert werden, anämische Gangrän eintritt; dasselbe Resultat ergibt die entsprechende Kompression der Mesenterialgefäße. Daraus schließt eben Sprengel, daß die Verstopfung von Vene oder Arterie zur Blutabsonderung ins Darmlumen, Darmwand und freie Bauchhöhle führt, während die Verlegung von Arterie und Vene anämische Gangrän zur Folge hat. Leider wurde bei den bisher beschriebenen Fällen auf diese Unterscheidung nicht geachtet und würde erst die weitere Beobachtung von Fällen von Mesenterialgefäßthrombosen die Richtigkeit dieser Ausführungen bestätigen können.

Wenn nun auch die Ansicht Borszekys richtig ist, daß Blutung nur bei einem Teile dieser Erkrankungen auftritt, so möchte ich diesem Symptom schon deshalb mehr Beachtung schenken, weil ja nur die Blutung uns auf die Möglichkeit einer Gefäßthrombose aufmerksam macht, während fast alle Fälle von anämischer Gangrän ohne Blutung als Ileus aufgefaßt wurden, und wahrscheinlich auch in Zukunft in vivo, bezw. vor der Operation nicht richtig diagnostiziert werden dürften.

Daß mitunter die Blutung so im Vordergrund stehen kann, daß sie uns das Bild eines Magengeschwüres vortäuschen kann, beweist ein Fall aus der medizinischen Abteilung des St. Johannspitales in Salzburg, der mir noch heute gut in Erinnerung ist.

Es war vor 6 Jahren (leider steht mir keine Krankengeschichte zur Verfügung) als ein Mann mit schwerer Hämatemesis, Abgang von Blut aus dem Darme und subnormaler Temperatur ins Spital gebracht wurde; der Patient starb noch am selben Tage unter Kollaps-Erscheinungen. Dit Sektion ergab anstatt des erwarteten Ulcus ventriculi eine hảmorrhagisehe Infarzierung mehrerer Dünndarmschlingen.

Es standen hier die peritonealen Anzeichen ganz im Hintergrund. Übrigens steht dieser Fall nicht vereinzelt in der Literatur da, indem mehrere Autoren ausdrücklich hervorheben, daß Blutung und Kollapserscheinungen die einzigen Symptome dieser Erkrankung darstellten.

Gelten so schon die zwei Hauptforderungen Kußmauls für die Diagnosenstellung: "Mesenterialgefäßthrombose", nämlich Darmblutung und Peritonitis, nur in beschränktem Maße, so ist auch die dritte Forderung: "Nachweis der Quelle der Embolie" nicht in allen Fällen notwendig. Über die damit in Zusammenhang stehende Frage der Ätiologie dieses Leidens werde ich später ausführlich 
sprechen und erwähne nur, daß auch in unserer Krankengeschichte eine bestimmte Ursache für die Thrombose nicht gefunden werden kann.

Was endlich das Sinken der Temperatur betrifft, so steht wohl dieses Symptom in innigstem Zusammenhang mit der Schwere der Blutung und wird daher in den meisten Fällen anämischer Gangrän fehlen, bezw. als terminales Anzeichen aufzufassen sein. In unserem Falle hatte die Patientin bei der Aufnahme 37,5. Interessant ist die schon früher erwähnte Besserung am 2. Tage. Die Morgentemperatur betrug nur 36,8; auch das Erbrechen hatte aufgehört. Ob nun dieses Sinken der Temperatur anf den immerhin bedeutenden Blutverlust zurückzuführen ist, läßt sich wohl schwerlich mit Sicherheit ausschließen; ich glaube jedoch nicht daran, da der Puls früh 80 betrug und kuäftig war. Anders der Zustand nachmittags: die T'emperatur steigt auf 38, der Puls schnellt auf 100, ja abends sogar auf 110 empor, die peritonealen Symptome setzen neuerdings ein. weshalb ich mich am 3. Tage zur Laparotomie entschloß. Es ist nun schwer verständlich, warum am 2. Tage früh die Krankheitserscheinungen wie verflogen waren, um dann nach weiteren 6 Stunden aufs neue zu beginnen. Auch Reitzenstein, macht, wie schon erwähnt, auf diesen Umstand aufmerksam. Dieses paradoxe Verhalten läßt sich meiner Ansicht nach nur dadurch erklären, daß man die Symptome des Ileus, der durch die plötzliche Infarcierung des Darmstückes bedingt ist, strenge von den Folgeerscheinungen der beginnenden Peritonitis scheidet. In den meisten Fällen kommt es zu einer Bauchfellentzündung überhaupt nicht, weil der Patient stirbt, bevor noch die Darmserosa mit von der Gangrän befallen ist. So betont z. B. Deladour ausdrücklich, daß bei der Obduktion seines Falles keine Peritonitis nachweisbar war. Hier war es wohl ausschließlich der Ileus und in weiterer Folge die Stercorämie, der die Patienten erliegen. In unserem Falle scheint aber der Ileus, der die nächste Folge der Thrombose darstellt, sich wieder gelöst zu haben, was ja begreiflich ist, da das exstirpierte Darmstück fast für Fingerdicke durchgängig war; erst als die durch den hämorrhagischen Infarkt bedingte Ernährungsstörung auch die Darmserosa ergriff, konnten die Bakterien ilneen Weg durch die Darmwand finden und kam es zur Ausbildung einer Peritonitis fibrinosa, die in der Temperatursteigerung, im Hinaufschnellen des Pulses ihren Ausdruck fand. Ich befinde mich mit dieser Auffassung in Übereinstimmung mit Deckart, der anf (irund eines Falles aus dem Allerheiligenspitale in Breslau zu dem 
Schlusse kam, daß die nächste Folge der Verstopfung der Mesenterialgefäße der Ileus paralyticus darstellt, während die Peritonitis erst sekundär hinzutritt. In dem von ihm mitgeteilten Falle war nur ein zirkulärer hämorrhagischer Infarkt vorhanden und doch stellte sich dieser als vollständiges Passagehindernis dar. Er erklärt diese Erscheinung damit, daß an der infarcierten Stelle die Kontraktion des Darmrohres nicht möglich ist und so die Ingesta nicht weiter getrieben werden können; es kommt zur vollständigen Stagnation des Darminhaltes, zum Ileus paralyticus und erst sekundär zur Peritonitis. Die Kontraktionsunmöglichkeit an der Stelle des Infarktes sei durch die Schädigung der Darmmuskulatur infolge Kohlensäure-Intoxikation bedingt; letztere ist eine Folge der venösen Stauung.

Nicht immer führt jedoch der Ileus paralyticus als solcher zum Tode, manches Mal, wie auch in unserem Falle, kann man ein Aufhören desselben beobachten. Daß dies nicht allzu selten ist, beweisen jene Fälle, in welchen auf einen akuten Beginn mit Erbrechen, Windverlaltung usw. ein ruhigeres Stadium folgt; allerdings ist diese Besserung nur eine trügerische, indem nach wenigen Stunden, höchstens Tagen, die Erscheinungen der Peritonitis in den Vordergrund treten.

Nach dem Vorhergesagten ist es allerdings nicht recht verständlich, warum in unserem Falle, da doch der Infarkt eine Ausdehnung von 30 bis $40 \mathrm{~cm}$ hatte, es zu einer Besserung kommen konnte, während im Falle Deckart der Infarkt sich ringförmig nur auf 2 bis 3 cm erstreckte und dennoch zum Tode führte. Ich bin natürlich nicht in der Lage, auf Grund dieser einzigen Beobachtung eine einwandfreie Erklärung dafür zu geben, glaube aber, daß es nicht so sehr auf die Ausdehnung des Infarktes, als vielmehr auf die Art der Thrombenbildung ankommt. Die Verschiedenheit des Beginnes dieser Erkrankung bald akut, bald chronisch, die geringe Einheitlichkeit des Symptomenkomplexes, bei gleichem pathologisch anatomischen Befund, läßt den Schluß zu, daß die Wirkung der Thrombose von verschiedensten Faktoren, die wir vielleicht niclit alle kennen, abhängt und daher keine einheitliche sein kann. So sind z. B. die Schnelligkeit des Entstehens des Thrombus, die mehr oder minder vollständige Verstopfung von Gefäßen, das eventuelle Vorhandensein von Kollateralen bestimmend auf den Grad der Ernährungsstörung. Dementsprechend braucht die Schädigung der Darmmuskulatur durch die Kohlensäure-Intoxikation keine derartige zll sein. daß sie zur vollständigen Kontraktionsunmöglichkeit führt. 
Hin durch Oper. geheilter Fall v. Thrombose im Geb. der Vena mes. sup. 87

das Passagehindernis wird, wenn auch schwer und langsam, von den herandrängenden Ingesta überwunden, der Ileus ist beseitigt. Bei der soeben erwähnten Verschiedenheit der Symptome wird es uns nicht überraschen, daß manchmal die Erkrankung statt mit Ileus mit blutigen Diarrhöen beginnt, ein Symptom, das ebenfalls die Folge der Ernährungsstörung darstellt.

Haben wir so die Symptomatologie genügend erörtert, so (lrängt sich uns als nächste Frage auf: führt die Mesenterialgefäßthrombose stets zur Infarcierung bzw. Gangrän? In dieser Hinsicht wird uns besonders der bekannte Fall Robsons interessieren, wo es nach Unterbindung der Vena mesenterica superior, die durch eine Verletzung notwendig wurde, zu glatter Heilung kam. Also trotz Verhinderung des Abflusses keine Gangrän, ja nicht einmal Infarcierung und bei Thrombose einer Nebenvene Infarkt, Ileus und meist auch tödlicher Ausgang. Polya glaubt, daß es nur dann zu Ernährungsstörungen kommt, wenn die Thrombosierung bis in die kleinsten Verzweigungen reicht, da ja sonst durch Anastomosen zwischen dem Gebiete der Mesenterica superior und inferior der Rückfluß ermöglicht wird. Auch in unserem Falle fand man die Thrombose auch in den kleinsten Nebenvenen vor. Leider konnte ich in den in der Literatur verzeichneten Fällen über die Ausdehnung der Thrombose keine bestimmten Angaben eruieren, meist wurden nur vereinzelte Thromben gefunden.

Nun ist aber noch ein anderes Moment zu berücksichtigen. Auch bei jeder incarcerierten Hernie kommt es, worauf Tietze aufmerksam macht, zur Venenthrombose und trotzdem finden wir keinen Bluterguß ins Darmlumen oder in die freie Bauchhöhle und nach Lösung der Einklemmung kann sich der Darm wieder erholen. Dieses verschiedene Verhalten erklärt Deckart damit, daß er für das Entstehen des Infarktes als auslösendes Moment die Schädignng der Darmmuscularis durch Enteritiden annimmt. Diese Erklärung ist schon deshalb nicht für alle Fälle stichhaltig, weil die anamnestischen Angaben über durchgemachte Darmkatarrhe unzuverläßlich sind, bezw. auch nicht in allen Fällen zutreffen. Ich glaube, daß man lierfür noch andere Momente heranziehen muß. Meiner Ansicht nach dürften dabei infektiöse Vorgänge und in weiterer Folge Veränderungen der Gefäßwände die Hauptrolle spielen; denn daß die Thrombose als solche nicht zum Infarkt führen muß, beweist eben das Verhalten bei der Incarceration. Nun ist es aber eine allgemein bekannte Tatsache, daß die Thrombose mit Gefäßverändernng in Zusammenhang steht. Bei der In- 
carceration jedoch ist die Gefäßwand als solche, da es sich bei der Einklemmung um ein zufälliges Ereignis handelt, meist intakt, die Thromben sitzen nicbt so fest und werden nach Lösung der Incarceration wieder weitergeschwemmt. Anders bei der Thrombose der Mesenterialgefäße als selbständiger Erkrankung. Hier kommt es zur Schädigung des Gefäßendothels meist durch Einwanderung von Bakterien, wie in Polyas Fällen oder durch andere noch später genauer zu erörternde Ursachen. Infolge der Veränderung der Gefäßintima kommt es zum Festsitzen des Thrombus, der dann dem Blutstrom festen Widerstand leistet und in weiterer Folge zur Infarcierung. $O b$ nun diese Ansicht für alle Fälle Geltung hat oder nicht, müßte die mikroskopische Untersuchung der thrombosierten Gefäße entscheiden; leider stand mir dieselbe nicht zur Verfügung und muß ich daher die Bestätigung dieser Ansicht der Untersuchung weiterer Fälle anheim stellen.

Bevor ich zur Besprechung der Ätiologie übergehe, möchte ich darauf hinweisen, daß unter den Thrombosen der Mesenterialgefäße die Embolie der Arteria mesenterica superior weit überwiegt. Kölbing konnte 58, Polya sogar 67 Fälle hiervon zusammenstellen, Deckart spricht von 46. Im Gegensatz hiezu ist die Thrombose der Vena mesenterica superior bedentend seltener. Kölbing berichtet über 11 Fälle, Polya sogar über 24 und fügt einen 25. hinzu, so daß mit Heranziehung meines Falles bis heute 26 publiziert sind. Noch seltener ist die Thrombose der Arterie und Vena mesenterica inferior. Deckart erwähnt 6 Fälle von "Thrombosis arterie mes. inferioris", Köster schildert 3 Fälle ron Thromb. ven. mes. inferior.

Einen Fall von Thrombose der Vena lienalis erwähnt derselbe Autor, wogegen Sachs über Pfortaderthrombose berichtet. Die von Deckart beschriebenen 6 geheilten Fälle lassen sich naturgemäß in eine dieser Gruppen nicht einreihen. Ich will mit der genauen Beschreibung all dieser Fälle nicht ermüden, da sie in den Abhandlungen von Deckart und Kölbing ohnedies ihre Würdigung erfahren haben und gehe sogleich zur Besprechung der Ätiologie über.

Hier möchte ich vor allem auf die Unterscheidung der Embolie der Mesenterialarterien und der Thrombose der Gekrösevenen hinweisen, da bei ersterer vor allem Zirkulationsstörungen das auslösende Moment darstellen. So wurde 3 mal Endokarditis, 3 mal Herzfehler ohne genaue Angabe, 1 mal Atheromatose und 1 mal Herzdegeneration als Ursache hingestellt. Bei den Fällen von Venen- 
thrombose als vermutliche Ursache: Thyphoid (Köster), Thrombophelitis der Vena saphena (Mignon und Doppler) Enterocolitis (Köster), nach Incarceration (Elliot); also, wie wir sehen, vorwiegend infektiöse Ursachen. Zu diesen Fällen kommt noch, wie uns Polya gezeigt hat, die Perityphilitis als Ursache der Erkrankung. Schon Deckart verweist auf den möglichen Zusarnmenhang dieser beiden Leiden, ohne jedoch mit Bestimmtheit denselben annehmen zu wollen. Erst Polya brachte 4 Fälle von Thrombophlebitis als Folge der Appendicitis, 2 Fälle hiervon verliefen allerdings unter den Symptomen der Pyämie, während die beiden anderen auch das klinische Bild der Mesenterialgefäßthrombose boten. Auch Payr ränmt den entzündlichen Vorgängen in der Ätiologie dieser Erkrankung einen hervorragenden Platz ein, kommt aber auf Grund der von ihm beobachteten Fälle von Thrombosenbildung während dor Operation (Vortrag Payrs am Chirurgen-Kongreb 1904) zu dem Schlusse, daß noch andere Ursachen für die Entstehung dieses Leiden's vorhanden sein müssen. Es waren nämlich gelegentlich zweier Bruchoperationen Thrombosen in den Darmvenen entstanden, die derart rasch auftraten, daß in beiden Fällen die Resektion der entsprechenden Darmschlinge notwendig wurde. Payr nimmt als Ursache der Venenthrombose neben den schon früher erwähnten entzündlichen Prozessen, mechanische Momente, die durch Kompression der Gefäße zur Zirkulationsstörung führen, sowie endlich auch rein traumatische Kontusion an. Bei seinen Fällen dürfte die Veränderung in den Zirkulationsverhältnissen durch die Operation die Ursache für die Thrombosierung abgegeben haben. Es ist begreiflich, daß bei "blander Embolie“ die Prognose eine günstigere ist als bei infizierter. Dem von diesem Autor hervorgehobenen Zusammenhang zwischen Operation und Thrombose begegnen wir auch bei Deladour, wo 23 Tage nach einer Splenektomie der Exitus unter Kollapserscheinungen eintrat und die Obduktion eine Embolie der Mesenterialvenen aufdeckte.

Was bei unserem Falle die Ursache der Erkrankung war, ist schwer zu sagen. Immerhin läßt die vor $1 \frac{1 / 4}{4}$ Jahre durchgemachte Beckenbindegewebsentzündung den Schluß zu, daß auch hier entzündliche Prozesse zu dem verhängnisvollen Leiden geführt haben.

Was die Prognose der Erkrankung betrifft, so ist sie, sowohl nach chirurgischen als auch internen Statistiken äußerst schlecht. So führt Kölbing von 58 Fällen 8 Spontanheilungen an; das wäre eine Mortalität von $86 \%$. Deckart berichtet über 6 Heilungen ohne Operation. Diesen schlechten internen Erfolgen gegenüber 
erscheint die chirurgische Statistik immerhin noch günstiger. Sprengel rechnet anf 14 Fälle 3 Heilungen, das sind $71 \%$ Mortalität. Kölbing auf 11 Fälle 2 Heilungen, das sind $81 \%$ Mortalität und endlich Lindner anf 11 Operationen 3 günstige Erfolge, also $72 \%$. Die 3 geheilten Fälle sind die von Elliot, Sprengel und Kölbing, auf die ich später noch zurückkommen werde. Rechnen wir zll diesen erwähnten Fällen noch den meinigen hinzll. und berücksichtigen wir die von Polya berichteten 2 operierten Fälle mit tödlichem Ausgang, so treffen auf 17 Operationen 4 Heilungen, das ist $761_{2}^{\prime} \%$, Mortalität.

Nun gebe ich natürlich ohne weiteres zll, daß der Vergleich der internen und chirurgischen Behandlung schon deshalb nicht einwandfrei ist, da bei letzterer die Erfahrung noch zn gering ist, möchte aber, wie ich später noch ausführen werde, darauf hinweisen, daß die für die Operation in Betracht kommenden Fälle. das sind die mit Ilenserscheinungen einhergehenden, von vornherein die schweren sind, während die mehr langsam einsetzenden einen leichteren Verlauf zu nehmen pflegen. Auch läßt sich gewiß bei besserer Auswahl der Fälle die Mortalität der chirurgischen Eingriffe herabsetzen.

Bevor ich die Frage über Zweckmäßigkeit und Indikationsstellung der Operation zu beantworten versuche, will ich im kurzen die mir aus der Literatur bekannten 3 Fälle mit günstigem Ausgang besprechen.

1. Fall Elliot: 25 jihriger Mann, 10 Monate früher eingeklemmter Bruch, der auf Taxisversuche zurückging. Beim Ballspiel traten plötzlich heftige Leibschmerzen mit Erbrechen verbunden auf. Es bestand kein Meteorismus, in der Cöcalgegend wurstförmige Geschwulst fühlbar. Operation: ein gangränöses Darmstück in der Lange von 48 Zoll wird reseziert, hierauf ein Anus practernaturalis angelegt. 14 Tage später Beseizigung des letzteren, Heilung.

2. Fall Sprengel: 38 jahriger Mann plötzlich mit Erbrechen und Schmerzen im Leib erkinankt. 6 Tage später Aufnahme ins Krankenhaus. Puls 80-84. P. macht keinen scliwerlkranken Einlruck.

In der linken Lumlalgegend Dämpfung; klinische Diagnose: Obdurations-Ileus, linksseitiges Exsudat, keine freie Peritonitis. Operation einige Stunden nach der Aufnahme. Laparotomie in der Medianlinie, in ıler Bauchhöhle rotbraune Flüssigkeit, Eventeration, gangränöses Darmstück von graubrauner Farbe, $10 \mathrm{~cm}$ lang, $30-40 \mathrm{~cm}$ von der Valvula Bauhini entfernt, bei der Resektion fliegt Darminhalt ins Abdomen. Anus praeternaturalis. Nach Einheilung desselben 2. Operation. Heilung.

3. Fall Kölbing: 31 jährige Frau, stets gesund. Am 24. VIII. 1901 Partus. Am 16. IX. nach mehrtägigen Diarrhöen Schmerzen im 
Ein durch Oper. geheilter Fall v. Thrombose im Geb. der Vena mes. sup. 91

Leib; am 19. IX. Erbrechen, am 21. IX. starker Meteorismus, Puls 125, klein, auf Einlauf kein Blutabgang, in der leocöcalgegend geblähte Darmschlinge fühlbar. Laparotomie: derb infiltrierte Jejunumschlinge $50 \mathrm{~cm}$ gangränös, Mesenterium blutig imbibiert. Resektion nach Verschluß der beiden Enden, hintere Gastronterostomia antecolica. Fall geheilt.

Den 2 letzten Fällen, sowie dem meinigen ist die verhältnismäßig geringe Ausdehnung der Gangrän gemeinsam und darin liegt wohl der Hauptgrund für den günstigen Ausgang. Vergleichen wir hiermit die Fälle von Operation mit tödlichem Ende, so trägt für den schlechten Erfolg entweder wie bei Köster die kolossale Ausdehnung der Gangrän die Schuld oder der Umstand,; daß der Allgemeinzustand bereits ein derartiger war, daß die Patienten der Chokwirkung der Operation erlagen. Es ist daher in erster Linie zu fordern, daß man sich nur dann zu einem Eingriff entschließe, wenn man einigermaßen Aussicht auf Erfolg hat; wir werden uns daher zu fragen haben, ob wir nicht schon vor der Operation imstande sind, uns von der Ausdehnung der Erkrankung ein klares Bild zul machen. Hier möchte ich auf den schon früher erwähnten Nachweis einer wurstförmigen Resistenz aufmerksam machen, die dann bei der Laparotomie genau der Stelle und Länge des infarcierten Darnıstückes entsprach; dieses Symtom wird allerdings nur bei Fehlen von Meteorismus zu beobachten sein. Haben wir uns über die Größe des infarcierten Stückes einmal orientiert, so wird die zweite Frage an uns herantreten: wann ist der günstigste Augenblick zur Operation? Soll man sofort operieren, oder, wie in unserem Falle, zunächst noch abwarten? Ich möchte hier auf die schon früher erwähnte Unterscheidung der Symptome in solche, die anf den Mens paralyticus zurückzuführen sind und solche, die der sekundär anftretenden Peritonitis angehören, zurückkommen.

Gegen die Operation im ersten Stadium sprechen schwer wiegende Bedenken, da ja, wie auch Deckart betont, bei Jleus paralyticus nicht operiert werden soll. In unserem Falle aber lagen die Verhältnisse anders; die lleuserscheinungen gingen am zweiten Tage zurück und erst die am selben Abend einsetzenden peritonealen symptome ließen uns mit der Operation nicht länger zögern, und daß wir mit diesem Standpunkt recht hatten, zeigt wohl der Erfolg; denn ich glaube kaum. daß ohne Operation eine Heilung möglich gewesen wäre.

Nun lehnt aber die Mehrzahl der Autoren die Operation bei Thrombose aus dem so eben angeführten Grunde, vor allem aber wegen der Schwere des Eingriffes ab. So glaubt selbst Sprengel. 
der doch auch über einen geheilten Fall berichtet, sich unter dem. Eindrucke der enormen Schwierigkeit der Operation gegen dieselbe aussprechen zu müssen. Dazu kommen die schlechten Erfahrungen, die von anderen Operateuren gemacht wurden. Auch ich will die Gefährlichkeit des Eingriffes nicht leugnen, da der Verlauf meines Falles durchaus kein glatter war. Schon bei der Operation floß aus dem geblähten zentralen Ende trotz größter Vorsicht Darminhalt heraus. Diese Gefahr ließe sich allerdings verringern, wenn man dem Vorschlage eines Operateurs folgend, nach Durchtrennung den Inhalt in ein Schälchen fließen lielse und dann erst zur Anastomose schritte. Im weiteren Verlaufe sind vor allem 2 Störungen zu verzeichnen. 1. Bildung einer Darmfistel; dieselbe kann mit der Tamponade' in Zusammenhang stehen oder, was ich glaube, durch Ernährungsstörungen bedingt sein, die ihren Grund in Zirkulationsstörungen im zurückgelassenen Mesenterium finden. Deshalb würde ich das nächste Mal die Resektion weiter im Gesunden vornehmen. Die zweite noch gefährlichere Störung war der erst am 21. Tage auftretende Ileus; derselbe war, wie die sofort ausgeführte Laparotomie zeigte, auf eine Knickung eines Darmstückes infolge Adhäsionsbildung zurückzuführen. Da die Lösung der Verwachsungen infolge Brüchigkeit der Darmwand unmöglich war, führte ich die Euterostomie ans, wobei ich durch den leichten Zug am zentralen Darmstück eine Streckung und dadurch Aufhebung der Knickung erhoffte. Tatsächlich schwanden die Ileuserscheinungen sofort und auch die Darmfistel schloß sich rasch. Der weitere Verlauf war infolge der Adhäsionen durch hartnäckige Obstipation getrübt. Schließlich gelang es uns, auch dieser Herr zu werden, worauf sich die Patientin schnell erholte.

Trotz der enormen Schwierigkeit, die dieser Fall bot, möchte ich dennoch unter gewissen Einschränkungen für die Operation eintreten, und zwar aus dem einfachen Grunde, weil wir sonst der ganzen Erkrankung machtlos gegenüberstehen. Andererseits sehen wir auch bei einer bereits vorgeschrittenen Gangrän (Fall Elliot) Heilung durch Operation eintreten.

Ich glaube meinen Standpunkt dahin präzisieren zu können, daß man nicht operieren soll bei Kollaps. bei Allgemeinperitonitis, und nicht sofort, solange die symptome des lleus paralyticus andanern. Vor allem soll man sich soweit als möglich über die Ausdehnung der vermutlichen Gangrän zu orientıeren suchen, wie es ja in unseren, suwie anderen Fällen tatsächlich möglich war. Es ist ja klar, 
Ein durch Oper. geheilter Fall v. Thrombose im Geb. der Vena mes. sup. 93

daß bei großer Ausdehnung der Erkrankung die Aussichten der Operation minimale sind und deshalb können die tödlich endenden Fälle von Sprengel und Köster zur Beantwortung der Frage, was die Operation leisten kann, nicht herangezogen werden.

Die eigentliche Indikation zum sofortigen Eingriff gibt meiner Ansicht nach der Beginn der peritonealen Symptome: Ansteigen derTemperatur, Pulsbeschleunigung. Hier wäre weiteres Abwarten von größter Gefahr. Unter diesen Voraussetzungen glaube ich für die Operation eintreten zu können und es wäre zum Schlusse noch die Frage der Operationswahl zu besprechen.

Die meisten Autoren wählten den Anus praeternaturalis, weil diese Operationsart leichter ausführbar und ungefährlicher als die Anastomosenbildung ist. Der Fall Kölbing hat nun gezeigt, daß auch letztere zur Heilung führen kann; ich möchte aber an der Hand meines Falles besonderen Wert darauf legen, daß die Resektion möglichst weit von der Demarkationslinie vorgenommen wird. um nicht Störungen im Heilungsverlauf ausgesetzt zu sein. Ich glaube, wenn die Anastomose möglich und nicht allzu schwer ist, und man sicher ist, weit im Gesunden zu sein, so ist sie der Anlegung des künstlichen Afters, besonders bei hochgelegener Gangrän, vorzuziehen, nur mülste die Vereinigung, worauf auch Lindner hinweist, von Seite zu Seite erfolgen, also so wie in unserem Falle.

Ich schließe mit der Überzeugung, daß bei genaner Indikationsstellung sich die chirurgische Mortalitätsziffer auch bei dieser Erkrankung noch weiter verringern wird zum Wohle der armen Patienten.

Nachtrag: Nach Vollendung dieser Arbeit kam mir die Mitteilung Brunners über einen durch Operation geheilten Fall von septischer Thromabose der Mesenterialvenen zu.

In dem von ihm publizierten Falle handelt es sich um einen 63 jährigen Herrn, der am 18. VII. unter fieberhaften Erscheinungen, verbunden mit Erbrechen erkrankte and Mitte August in die Anstalt aufgenommen wurde. (Die Temperaturen schwankten zwischen $37^{\circ}$ und $40^{\circ}$.) Auf Grund dieser Symptome, verbunden mit dem Nachweis eines Tumors links vom Nabel, wurde die Diagnose auf Cad. flexur. sigmoid. mit AbszeBbildung und septischer Phlebitis gestellt. Die Operation ergab eine Infarcierung eines $12 \mathrm{~cm}$ langen Darmstückes infolge zahlreicher Thromben in dem zugehörigen Mesenterium. Es wurde die Resektion des Darmstückes und die Anastomose mittels Murphyknopf ausgeführt. Brunner nimmt als Ätiologie dieses Falles eine vom Darm aus aufsteigende Infektion der Venen an. 
Von den in der Arbeit angeführten Literaturangaben sei erwähnt, daß Brunner 125 Fälle von Thrombose der Gekrösearterien zusammenstellen konnte, von welchen 26 operiert wurden, hiervon nur 2 mit glücklichem Ausgang. Von den 89 in der Literatur bekannten Venenthrombosen wurden 31 operiert, hiervon 4 geheilt, mit Brunners Fall 5, mit meinem 6. Es beträgt somit die Martalität derzeit 80,7 Proz.

Ferner möchte ich zum Schlusse noch auf den Aufsatz in der deutschen Zeitschrift für Chirurgie, 90. Bd., Heft 1-3 von Marek: .,̈̈ber die Folgen von Verschluß der Gekrösearterien" hinweisen der mir leider nur im Referat der Münchner med. Wochenschrift Nr. 44, Jahrgang 1907 zur Verfügung steht. Der Verfasser kommt auf Grund von 18 Versuchen an Hunden zu folgenden Schlusse: Die vollständige Absperrung des arteriellen Zuflusses verursacht anämische Gangrän, die Verlegung von Arterien, bezw. Venen gesondert oder gleichzeitig bei ungenügendem Kollateralenkreislauf hingegen hämorrhagischen Infarkt. Das Bild des letzteren gestaltet sich verschieden nach dem Grade der Stromverlangsamung und der Höhe des intrakapillaren Druckes; ein Ergebnis, das in teilweisem Widerspruche mit den Angaben Sprengels steht.

\section{Literaturverzeichnis.}

1. Zentralblatt für Chirurgie 1906, Nr. 14: Lindner, Thrombose der Mesent.-Gefäße. Ref.'

2. Zentralblatt für Chirurgie 1906, Nr. 38: Gull, Thrombos of mes. vessels. Bristol med. Chirurg. journal No. 2. Ref.

3. Zentralblatt für Chirurgie 1905, Nr. 10: Mignon et Doppler, Thrombophlebite meseraïque. Ref.

4. Zentralblatt für Chirurgie 1905, Nr. 30: Referat über Sprengels Vortrag am Kongreß der deutschen Gesellschaft f. Chir. 1905.

5. Zentralblatt für Chirurgie 1904: Referat über Payrs Vortrag am Kongreß der deutschen Gesellschaft f. Chir. 1904.

6. Zentralblatt für Chirurgie 1895, Nr. 46: Deladour, Thrombosis of the inesent. veins as a cause of death after splenectomie. Ref.

7. Zentralblatt für Chirurgie 1855, Nr. 13: W. Gull, Cas of probable Thrombosis of superior mesent. veins and renal veins. Ref.

8. Beiträge zur klin. Chirurgie 1902, Bd. 33, Nr. XIII: Dr. P. Kölbing, Beiträge zur Magen-Darm-Chirurgie. 1. Thrombose der Mesenterialgefäße.

9. Deutsche medizinische Wochenschrift 1905: Dr. Eugen Polya, Thrombophlebitis meseraica, eine verhängnisvolle Komplikation der Appendicitis. 
Ein durch Oper. geheilter Fall v. Thrombose im Geb. der Veua mes. sup. 95

10. Archiv fürklin. Chirurgie, Bd. 67, Nr. XXII: Prof. Dr. Sprengel, Zur Pathologie der Zirkulationsstörungen im Gebiete der Mesenterialgefäßre.

11. Mitteilungen aus den Grenzgebieten der Medizin und Chiurgie, Bd. 5, Nr. XVIII: Dr. P. Deckart, Über Thrombose und Embolie der Mesenterialgefäße. Ein Beitrag zur Lehre des Ileus.

12. Deutsche med. Wochenschrift 1898, Nr. 21: Dr. H. Köster, Zur Kasuistik der Thrombose und Embolie der großen Bauchgefäße.

13. Beiträge zurklin. Chirurgie, Bd. 31, Nr. XXVI: Dr Karl Borszeky, Ileus durch Embolie der Art. mes. sup.

14. Münchner med. Wochenschrift 1903, Nr. 6: Dr. A. Reitzenstein, Ileus infolge Thrombose der Vena mesent.

15. Deutsche med. Wochenschrift 1892, Nr. 20: Dr. Richard Sachs, Zur Kasuistik der Gefälerkrankungen. Kapitel Pfortaderthrombose.

16. Deutsche Zeitschrift f. Chir., 89. Bd., 5. u. 6. Heft, Nr. XVIII Dr. F. Brunner, Ein durch Operation geheilter Fall von septischer Thrombose der Mesenterialgefäße.

17. Münchner med. Wochenschrift 1907, Nr. 44: Ref. aus der Deutschen Zeitschr. f. Chir, Bd. 90, H. 1-3. Marek, Über die Folgen des Verschlusses der Gekrösearterien. 